\title{
Brain Cancer Cell-derived Exosomes Protect Scopolamine-In- duced Death of SH-SY5Y Neuron Cells
}

\author{
Minseo Lee \\ Cranbrook Kingswood, 39221 Woodward Ave, Bloomfield Hills, MI 48304, USA; gosyber@suwon.ac.kr
}

\begin{abstract}
Exosomes are the nano-sized extracellular vesicles secreted by most of the cell types. The role of exosomes in cells is to function as important intercellular messengers and contributors to both disease development and health. Many studies have discovered the role of exosomes in Alzheimer's disease (AD). Exosomes have been shown to induce apoptosis and neuronal loss. On the other hand, exosomes possess the ability to reduce brain amyloid-beta and to transfer neuroprotective substances between cells. However, the effect of exosomes derived from cancer cells has never been investigated. In this study, it was hypothesized that exosomes derived from cancer cells protect neurons from cell death because these exosomes are known to contain many cancer-promoting substances that proliferate cancer cells. This study found that exosomes derived from the A172 brain cancer cell line increase cell viability of differentiated SH-SY5Y neuron cells from scopolamine hydrobromide (SH)-induced cell death. Since cancer-derived exosomes have potential as therapeutic tools for $\mathrm{AD}$ with their neuroprotective effects, further studies are required to determine what substances inside the exosome protect the neuron cells from SH-induced cell death.

KEYWORDS: Biochemistry; Physiology; Exosome; Cancer; Neuron; Scopolamine Hydrobromide; Cell Death.
\end{abstract}

\section{Introduction}

Alzheimer's disease $(\mathrm{AD})$ is a type of dementia that interferes with memory, thinking, and behavior; it is a progressive disease that worsens over time. ${ }^{1}$ Early-stage Alzheimer's symptom entails having difficulty learning since it typically originates in the part of the brain that controls learning. ${ }^{1}$ Subsequently, Alzheimer's patients go through severe symptoms, such as behavioral changes, confusion surrounding dates and events, and difficulty speaking and swallowing. ${ }^{2}$ Two abnormal brain structures, plaques and tangles, are suspected of damaging nerve cells, and therefore leading to Alzheimer's. ${ }^{2}$ Plaques are deposits of protein fragment beta-amyloid that takes space between nerve cells, and tangles are twisted fibers of a protein called tau. ${ }^{3}$ Despite the common belief that Alzheimer's is reserved for the elderly, approximately 200,000 Americans have younger-onset Alzheimer's disease. ${ }^{4}$

$\mathrm{SH}-\mathrm{SY} 5 \mathrm{Y}$ is a human-derived cell line isolated from the bone marrow of a four-year-old female with neuroblastoma. ${ }^{5}$ SH-SY5Y cells can be differentiated from a neuroblast-like state into mature human neurons using retinoic acid (RA). ${ }^{5}$ The molecular mechanism of retinoic acid's role in neuronal differentiation has yet to be determined. However, a previous study hypothesized that it might regulate receptor tyrosine kinase-like orphan receptor ${ }^{1}$ (ROR1) for retinoic acid-induced differentiation. ${ }^{6}$ In this experiment, retinoic acid is used to differentiate $\mathrm{SH}-\mathrm{SY} 5 \mathrm{Y}$ into neuron cells.

Scopolamine hydrobromide (SH) causes cytotoxicity and downregulation of neuronal and glial cell markers. ${ }^{7} \mathrm{SH}$ blocks the activity of muscarinic acetylcholine receptor, which is frequently observed in those with $\mathrm{AD}$. Even though $\mathrm{SH}$ cannot induce the full range of deficit seen in patients with $\mathrm{AD}, \mathrm{SH}$ is often used to mimic the damage of $\mathrm{AD}$ in cell culture models. ${ }^{7}$
Therefore, $\mathrm{SH}$ is used to mimic the damage of Alzheimer's disease on differentiated $\mathrm{SH}-\mathrm{SY} 5 \mathrm{Y}$ in this study.

The exosome is an extracellular vesicle released from cells that carries proteins and nucleic acid materials, including all types of RNA. ${ }^{8}$ Cancer cell-derived exosomes also carry the biological contents of cancer cells but have different effects. ${ }^{9}$ The four effects of cancer cell-derived exosomes are the formation of metastatic lesions, the acquisition of the capacity to avoid attacks from immune cells, the acquisition of migratory and invasive capacity, and the enlargement of cancer tissue angiogenesis and cell proliferation. ${ }^{10}$ Also, cancer cell-derived exosomes are messengers of signals that involve pathogenesis, development, progression, and metastasis of cancer cells. ${ }^{10}$

In this investigation, the goal was to discover the effects of cancer-derived exosomes on cell viability of differentiated $\mathrm{SH}-\mathrm{SY} 5 \mathrm{Y}$ cells. It was predicted that these exosomes would increase the cell viability of neuron cells by delivering cancer cell-derived proteins and RNAs, which are known to enhance the cell cycle and inhibit apoptosis. Therefore, considering the close relationship between cancer cell-derived exosomes and cell proliferation and survival, the protective effect of cancer cell-derived exosome on cell viability of SH-induced neuronal cell death was examined.

\section{- Methods}

SH-SY5Y and A172 cell line culture:

SH-SY5Y and A172 cell lines were purchased from Korea Cell Line Bank (KCLB). The cells were maintained at $37^{\circ} \mathrm{C}$ in a $\mathrm{CO}_{2}$ incubator. Cells were trypsinized and fresh RPMI media (GIBCO) was provided every three or four days.

\section{Exosome isolation from cell culture media:}

From its cell culture media, the exosome of the A172 cancer cell was isolated. After harvesting $8 \mathrm{~mL}$ cell culture media of A172, it was mixed with $4 \mathrm{~mL}$ exosome isolation reagent (Sig 
ma). Then, the culture media/reagent mixture was incubated in $4{ }^{\circ} \mathrm{C}$ overnight. $1 \mathrm{~mL} 1 \mathrm{X}$ PBS (WELGENE) was used for resuspension. The isolated exosome is then stored in $-80{ }^{\circ} \mathrm{C}$ for long-term storage. Negative control exosome was prepared from RPMI 1640 cell culture media (i.e. treated the same but no cells added) to check if the media is completely depleted with any exosomes from cancer cells.

\section{Retinoic Acid (RA) treatment:}

The retinoic acid stock solution was made to have a concentration of $5 \mathrm{mM} .15 .022 \mathrm{mg}$ retinoic acid powder (SIGMA, R2625) was dissolved in $10 \mathrm{~mL}$ DMSO (JUNSEI, lab no. 201414005). After vortexing, the mixture of retinoic acid powder and DMSO, was filtrated of small floating matter by using a 0.22 filter on the tip of the injector. Final concentration of $5 \mathrm{mM}$ of RA was used to differentiate $\mathrm{SH}-$ SY5Y cells into neurons.

\section{Scopolamine hydrobromide (SH) treatment:}

Scopolamine hydrobromide stock solution was made to have a concentration of $50 \mathrm{mM}$. $109.5 \mathrm{mg}$ scopolamine hydrobromide powder (SIGMA-ALDRICH, PHR1470) was dissolved in $5 \mathrm{~mL}$ DDW (BIONEER, C-9011). The working condition for the scopolamine hydrobromide solution was decided as $2 \mathrm{mM}$, after examining and comparing the effect of five different concentrations of scopolamine hydrobromide.

\section{Western blot analysis:}

Whole-cell lysates were prepared using Passive Lysis Buffer purchased from Promega (Madison, WI). The cell extracts $(50 \mu \mathrm{g})$ were loaded on a $15 \%$ sodium dodecyl sulfatepolyacrylamide gel for electrophoresis, and proteins were transferred to polyvinylidene fluoride membranes with a Mini Trans-Blot@ tank (Bio-Rad, Hercules, CA). The membrane was probed with antibodies against Flotillin-1 (ab41927, Abcam), CD63 (EXOAB-KIT-1, System Bioscience), and GM130 (cell signaling). All secondary antibodies (Santa Cruz Biotechnology, Dallas, TX) were incubated for $1 \mathrm{hr}$. Detection was performed using an Amersham ECL GST Western Blotting Detection Kit (GE Healthcare Life Sciences, Piscataway, NJ).

\section{Analyzing the total cell number and cell viability in \\ SH-SY5Y:}

The cells were trypsinized and afterwards isolated exosome and $\mathrm{SH}$ were incubated for $72 \mathrm{~h}$. The percentage of viable cells was measured by counting cells, which were stained by acridine orange/propidium iodide, an apoptosis indicator, with the $\mathrm{Lu}-$ na-FL Dual Fluorescence Cell Counter (Logos Biosystems).

\section{Statistical analysis:}

Data were expressed as the mean values \pm standard error and analyzed by Student's t-test using Prism 7 and Microsoft Excel. All statistical results were considered significant if $\mathrm{p}$-values were 0.05 or less.

\section{- Results and Discussion Differentiation of SH-SY5Y cells to neuron cells by retinoic acid:}

Several studies indicated important differences between undifferentiated and differentiated SH-SY5Y cells. ${ }^{11,12}$ When SH-SY5Y cells are undifferentiated, they appeared to be non-polarized and proliferate rapidly. ${ }^{12}$ When they are differ- entiated, the cells appeared to be branched and extend long with a decrease in cell proliferation. ${ }^{12}$
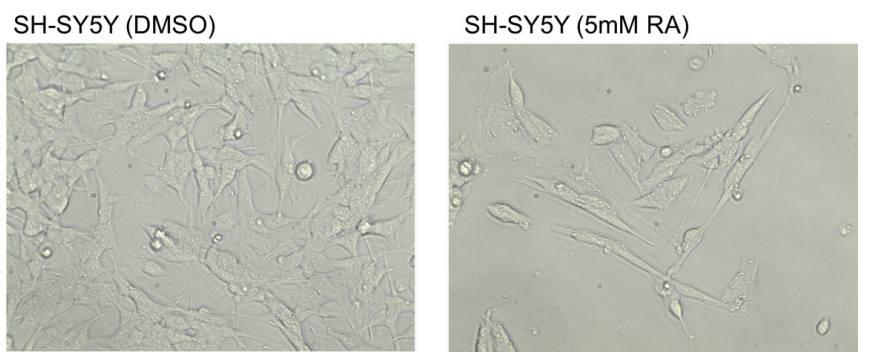

Figure 1: Images of $\mathrm{SH}^{-} \mathrm{SY}^{5} \mathrm{Y}$ cells treated with DMSO and $5 \mathrm{mM}$ Retinoic Acid (RA).

To confirm the differentiation of SH-SY5Y to neuronal cells by RA, we checked the changes in cell morphology and cell confluence. Figure 1 shows the change in cell morphology of SH-SY5Y cells after $5 \mathrm{mM}$ RA treatment. Compared to the SH-SY5Y that was treated only with DMSO, SH-SY5Y with $5 \mathrm{mM}$ RA displayed longer and narrower cell shape with fewer numbers of cells (Figure 1). This result indicates that RA successfully differentiated SH-SY5Y cells into neurons. However, the neuronal characteristics were not fully validated by the classical neuronal markers in this experiment.

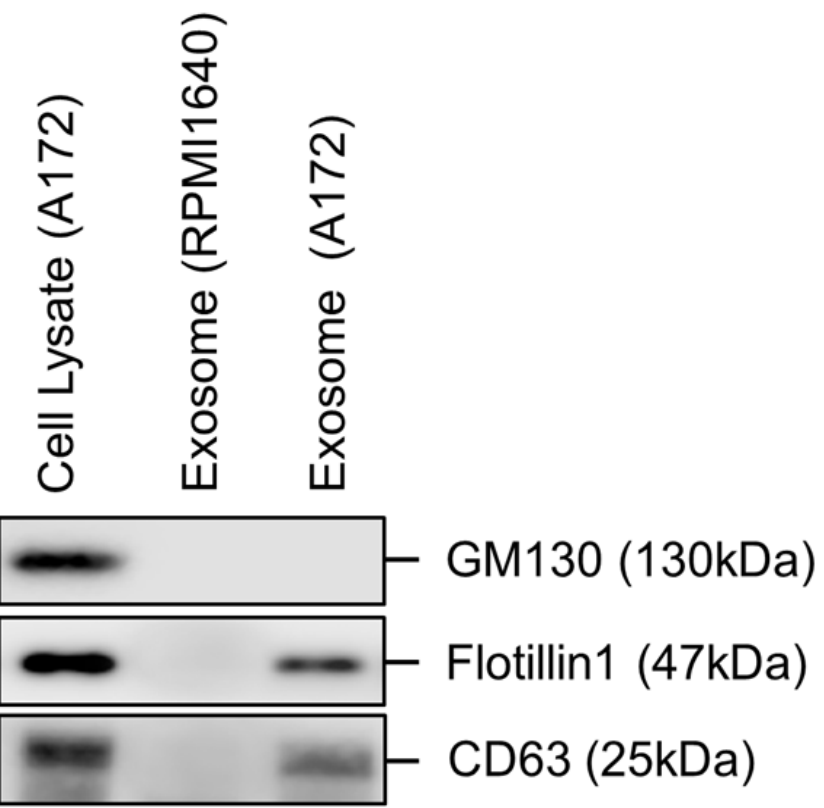

Figure 2: The protein expression level of GM130, Flotillin1, and CD63 analyzed by western blot. The protein size of the target proteins is indicated in $\mathrm{kDa}$.

\section{Exosome isolation from A172 cell culture media:}

Human A172 cell line was used to collect brain cancer cell-derived exosomes from cell culture media. The western blot was used in this experiment in order to make sure that the exosome was completely isolated in our experiment without any contamination of cell lysate. Three samples were analyzed in this experiment: cell lysate for positive control, exosome isolated from RPMI1640 cell culture media for the negative control to make sure the media is truly depleted of cancer-de- 
media (Figure 2). Figure 2 shows that the cell lysate contained all proteins, but the exosome isolated from RPMI 1640 media does not contain any proteins. It also demonstrates the presence of canonical exosome proteins (Flotillin1 and CD63) and the absence of Golgi protein GM130 from isolated A172 derived exosome (Figure 2). Taken together, the western blot demonstrated that A172 derived exosome produces high quality purified exosomes free from other cellular contaminants.

Protective effect of cancer cell exosomes on $\mathrm{SH}$-mediated neuronal cell death :

A
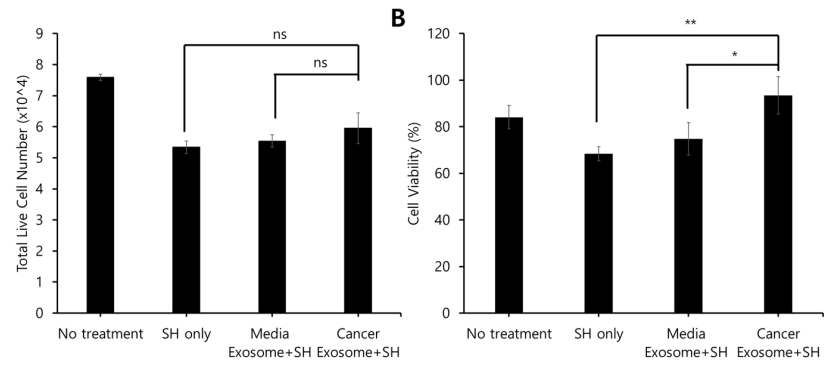

Figure 3: Effect of exosome pre-treatment on total cell number and cell viability of $\mathrm{SH}^{-} \mathrm{SY} 5 \mathrm{H}$. Three biological replicates were measured $(\mathrm{n}=3)$.

To confirm the protective role of cancer cell-derived exosomes on $\mathrm{SH}-\mathrm{SY} 5 \mathrm{Y}$ cell death driven by $\mathrm{SH}$, we pretreated exosomes on SH-SY5Y for $1 \mathrm{hr}$. Then, the cells were further incubated with $\mathrm{SH}$ for $24 \mathrm{hr}$ to induce cell death. To compare the effect of cancer cell-derived exosome on cell viability, we prepared four different groups of samples: no treatment, $\mathrm{SH}$ only, media exosome with $\mathrm{SH}$, and cancer cell-derived exosome with $\mathrm{SH}$. No treatment sample was used as a negative control that was not treated with $\mathrm{SH}$ and exosomes. The $\mathrm{SH}$ only sample was treated only with $\mathrm{SH}$ but not pretreated with any exosomes. Media exosome with $\mathrm{SH}$ sample was pretreated with RPMI1640-derived exosome and SH was post-treated. Cancer cell-derived exosome with SH sample was pretreated with cancer cell-derived exosome and $\mathrm{SH}$ was post-treated.

Figure 3 demonstrates the effect of cancer cell-derived exosome on SH-SY5Y cell number and cell viability. The cancer cell-derived exosome has no significant effect on total number of live cells (Figure 3A). However, cancer cell derived exosome significantly increased the cell viability compared to $\mathrm{SH}$ only sample and media exosome with SH sample (Figure 3B). Since this experiment only analyzes the early effect of cancer cell-derived exosomes on cell viability that the incubation time of both exosome and $\mathrm{SH}$ is limited to $25 \mathrm{hr}$., further experiments are needed to examine the change in cell viability for longer incubation times.

Exosomes are involved in the spread of 'toxic' proteins in neurodegenerative disorders such as AD, Huntington's disease (HD), and Parkinson's disease (PD). ${ }^{13,14}$ The mutated or misfolded proteins induce neuronal cell death. ${ }^{13}$ On the other hand, the role of exosomes on neuronal protection, nerve regeneration, and neuronal development have been studied, indicating the release of exosomes by neurons, microglia, and astrocytes. ${ }^{15}$ However, the role of exosomes derived from cancer cells on neuronal protection has never been investigated. In this study we found that brain cancer derived exosomes have neuroprotective effects against $\mathrm{SH}$-mediated cell death on differentiated neuronal SH-SY5Y cells. A previous study indicated that cancer derived exosomes from lung cancer cells inhibit cell apoptosis of normal lung fibroblast. ${ }^{10}$ Colon cancer cells secrete exosomes to promote self-proliferation by shortening mitosis duration. ${ }^{16}$ These studies suggested that exosomes from cancer cells delivered functional, transportable mRNA, miRNA, and oncogenic proteins and activated proliferation-related cellular pathways. Therefore, delivering these substances by cancer derived-exosome likely contributed to inhibiting neuronal cell death induced by SH. However, future studies are needed to confirm which molecules from the cancer-derived exosome protect the neurons.

$\mathrm{SH}$ is known to cause cell death through intrinsic apoptosis in neuronal cells. ${ }^{17}$ In addition, scopolamine decreased cell viability and MMP, and increased caspase-3 activation and PARP cleavage in SH-SY5Y cells. ${ }^{17}$ Therefore, it is possible that cancer derived exosomes may block the intrinsic pathway of apoptosis to protect SH-SY5Y cells. However, screening of apoptosis markers is needed to elucidate the molecular mechanism of neuroprotective effect induced by cancer-derived exosomes.

\section{Conclusion}

It was discovered that the cancer cell-derived exosomes have a protective role on neuronal cell death caused by SH. However, since our experiment was solely on the SH-SY5Y cell line, further experiments with different neuron cells are needed to confirm the role of cancer cell-derived exosomes. Since the research indicates that cancer-derived exosomes have potential as therapeutic tools for $\mathrm{AD}$ with its neuroprotective effect, further study is required to determine the substances inside the exosome that are responsible for the protection from $\mathrm{SH}$-induced neuronal cell death. It will also be necessary to identify the effect of cancer-derived exosomes from many different types of brain cancer cell lines for performing validation experiments.

\section{Acknowledgement}

I would like to thank Dr. Woo Rin Lee, my mentor from University of Suwon for his assistance and guidance in this project.

\section{- References}

1. Schachter, A. S.; Davis, K. L., Alzheimer's disease. Dialogues in clinical neuroscience 2000, 2 (2), 91-100.

2. Bature, F.; Guinn, B. A.; Pang, D.; Pappas, Y., Signs and symptoms preceding the diagnosis of Alzheimer's disease: a systematic scoping review of literature from 1937 to 2016. BMJ open 2017, 7 (8), e015746.

3. Chong, F. P.; Ng, K. Y.; Koh, R. Y.; Chye, S. M., Tau Proteins and Tauopathies in Alzheimer's Disease. Cellular and molecular neurobiology 2018, 38 (5), 965-980.

4. Mendez, M. F., Early-Onset Alzheimer Disease.

Neurologic clinics 2017, 35 (2), 263-281.

5. Kovalevich, J.; Langford, D., Considerations for the use of SH-SY5Y neuroblastoma cells in neurobiology. Methods in molecular biology 2013, 1078, 9-21.

6. 1lendula, A.; Fultang, N.; Peethambaran, B., Retinoic acid induces differentiation in neuroblastoma via ROR1 
by modulating retinoic acid response elements. Oncol Rep 2020, 44 (3), 1013-1024.

7. Bajo, R.; Pusil, S.; Lopez, M. E.; Canuet, L.; Pereda, E.; Osipova, D.; Maestu, F.; Pekkonen, E., Scopolamine effects on functional brain connectivity: a pharmacologi cal model of Alzheimer's disease. Scientific reports 2015, 5, 9748 .

8. Zhang, Y.; Liu, Y.; Liu, H.; Tang, W. H., Exosomes: biogenesis, biologic function and clinical potential. Cell \& bioscience 2019, 9, 19 .

9. Blackwell, R. H.; Foreman, K. E.; Gupta, G. N., The Role of Cancer-Derived Exosomes in Tumorigenicity \& Epithelial-to-Mesenchymal Transition. Cancers 2017, 9 (8).

10. Huang, J.; Ding, Z.; Luo, Q.; Xu, W., Cancer cellderived exosomes promote cell proliferation and inhibit cell apoptosis of both normal lung fibroblasts and nonsmall cell lung cancer cell through delivering alphasmooth muscle actin. Am J Transl Res 2019, 11 (3), 1711-1723.

11. Forster, J. I.; Koglsberger, S.; Trefois, C.; Boyd, O.; Baumuratov, A. S.; Buck, L.; Balling, R.; Antony, P. M., Characterization of Differentiated SH-SY5Y as Neuronal Screening Model Reveals Increased Oxidative Vulnerability. Journal of biomolecular screening 2016, 21 (5), 496-509.

12. Shipley, M. M.; Mangold, C. A.; Szpara, M. L., Differentiation of the SH-SY5Y Human Neuroblastoma Cell Line. J Vis Exp 2016, (108), 53193.

13. Bellingham, S. A.; Guo, B. B.; Coleman, B. M.; Hill, A. F., Exosomes: vehicles for the transfer of toxic proteins associated with neurodegenerative diseases? Frontiers in physiology 2012, 3, 124.

14. Soria, F. N.; Pampliega, O.; Bourdenx, M.; Meissner, W. G.; Bezard, E.; Dehay, B., Exosomes, an Unmasked Culprit in Neurodegenerative Diseases. Frontiers in neuroscience 2017, 11, 26.

15. Kalani, A.; Tyagi, A.; Tyagi, N., Exosomes: mediators of neurodegeneration, neuroprotection and therapeutics. Molecular neurobiology 2014, 49 (1), 590-600.

16. Ren, R.; Sun, H.; Ma, C.; Liu, J.; Wang, H., Colon cancer cells secrete exosomes to promote self-proliferation by shortening mitosis duration and activation of STAT3 in a hypoxic environment. Cell \& bioscience 2019, 9, 62 .

17. Puangmalai, N.; Thangnipon, W.; Soi-Ampornkul, R.; Suwanna, N.; Tuchinda, P.; Nobsathian, S., Neuroprotection of N-benzylcinnamide on scopolamineinduced cholinergic dysfunction in human SH-SY5Y neuroblastoma cells. Neural regeneration research 2017, 12 (9), 1492-1498.

\section{- Author}

Minseo Lee is a 12th-grade student in Cranbrook Kingswood Upper School. Since she is still a high school student, she hasn't decided on her college major yet. In college, she hopes to major in neuroscience with a biological approach. 